\title{
Herbal tea-induced hepatic veno-occlusive disease in China: a case description
}

\author{
Bo $\mathrm{Li}^{1}$, Fabao Gao ${ }^{2}$, Xinai Liu ${ }^{3}$, Junzheng $\mathrm{Pan}^{4}$, Lin $\mathrm{Liu}^{5}$ \\ ${ }^{1}$ West China School of Medicine, Sichuan University, Chengdu, China; ${ }^{2}$ Department of Radiology, West China Hospital, Chengdu, China; \\ ${ }^{3}$ Department of Radiology, Henan Province Hospital of TCM, Zhengzhou, China; ${ }^{4}$ Department of General Surgery, Henan Province Hospital of \\ TCM, Zhengzhou, China; ${ }^{5}$ Department of Radiology, Zhumadian Central Hospital, Zhumadian, China
}

Correspondence to: Fabao Gao. No. 1 Keyuan 4th Road, Wuhou District, Chengdu City, Sichuan Province, China. Email: gaofabao@wchscu.cn.

Submitted Jan 08, 2020. Accepted for publication Jan 07, 2021.

doi: 10.21037/qims-20-48

View this article at: http://dx.doi.org/10.21037/qims-20-48

\section{Introduction}

Hepatic veno-occlusive disease (HVOD) [also known as hepatic sinusoidial obstruction syndrome (HSOS)] occurs sporadically throughout the world (1-7). Several factors cause HVOD: the intentional or unintentional intake of pyrrolizidine alkaloid (PA)-containing products $(8,9)$. Tusanqi is used to improve blood circulation, dissipate blood stasis, and treat traumatic injuries. However, its roots contain PA, which can cause hepatotoxicity if consumed in large amounts or consumed incorrectly $(10,11)$. For this reason, Tusanqi is one of the main causes of HVOD in China.

\section{Case presentation}

A 27-year-old female was admitted to the Henan Province Hospital of Traditional Chinese Medicine (TCM) due to abdominal distention, fatigue, anorexia, and discomfort in the right flank for 2 weeks. For almost 1 year, her menstrual blood volume had increased, and bleeding was prolonged for no apparent reason. At her husband's request, she started drinking approximately 10 cups of herbal tea prepared by the family daily and consumed the tea for almost three months before developing worsening abdominal symptoms. Subsequent investigation revealed the tea contained Tusanqi.

The following findings were present; jaundice, abdominal distention with upper abdominal tenderness, hepatomegaly with the liver approximately $3 \mathrm{~cm}$ below the arcus costarum, splenomegaly, ascites with shifting dullness, and mild edema in the lower extremities.

Laboratory tests showed that the ALT (18 U/L) and AST (31 U/L) levels were within the normal range (0-40 U/L). However, total bilirubin $(57.6 \mu \mathrm{mol} / \mathrm{L})$, direct bilirubin $(27.5 \mu \mathrm{mol} / \mathrm{L})$, and total bile acid $(74.2 \mu \mathrm{mol} / \mathrm{L})$ were above the normal ranges of $0-23.0,0-6.8$, and $0-10.0 \mu \mathrm{mol} / \mathrm{L}$, respectively). Total protein $(48.2 \mathrm{~g} / \mathrm{L})$ and serum albumin $(26.8 \mathrm{~g} / \mathrm{L})$ were below the normal ranges of $60.0-87.0$ and 35.0-53.0 g/L, respectively. Moreover, serum prealbumin levels were significantly decreased $(28 \mathrm{mg} / \mathrm{L}$, normal range of $180-400 \mathrm{mg} / \mathrm{L})$. HBV-DNA data above the normal, HBsAg+, HBsAb-, HBeAg-, HBeAb+, HBcab-IgM+, and HCV-Ag-, HCV-Ab- (Table 1).

Abdominal Doppler ultrasound (US) examination showed that the liver was increased in size, and the capsule was smooth. The echo of the liver parenchyma was uneven, and no specific mass echo was seen. The hepatic veins were normal in shape, the diameter of the tube had become thinner, and no intervenous communication branch was seen. No thrombosis was found in the hepatic veins, and Colour Doppler Flow Imaging (CDFI) indicated a fine flow signal in the lumens. The portal vein system was not widened, and the mean blood flow velocity of the right portal vein was $0.19 \mathrm{~m} / \mathrm{s}$. The lumen of the inferior vena cava (IVC) had no obvious stenosis, and the blood flow direction was normal.

Abdominal CT images showed hepatomegaly, a diffuse uniform reduction in the liver parenchyma density, gallbladder wall thickening and blurring, gallbladder fossa effusion, and splenomegaly. Triphasic contrast-enhanced 
Table 1 Results of laboratory tests for the first hospitalization

\begin{tabular}{|c|c|c|c|c|}
\hline Inspection item & $\begin{array}{l}\text { Inspection } \\
\text { result }\end{array}$ & Mark & Unit & $\begin{array}{c}\text { Reference } \\
\text { value }\end{array}$ \\
\hline ALT & 18 & & $U / L$ & $0-40$ \\
\hline AST & 31 & & $U / L$ & $0-40$ \\
\hline TP & 48.2 & $\$$ & $g / L$ & $60.0-87.0$ \\
\hline ALB & 26.8 & $\$$ & $\mu \mathrm{mol} / \mathrm{L}$ & $35.0-53.0$ \\
\hline TBIL & 57.6 & $\#$ & $\mu \mathrm{mol} / \mathrm{L}$ & $0-23.0$ \\
\hline DBIL & 27.5 & $\#$ & $\mu \mathrm{mol} / \mathrm{L}$ & $0-6.8$ \\
\hline TBA & 74.2 & $\#$ & $\mu \mathrm{mol} / \mathrm{L}$ & $0-10.0$ \\
\hline GGT & 49 & \# & $U / L$ & $0-35$ \\
\hline $\mathrm{CHE}$ & 2.91 & $\$$ & $\mathrm{KU} / \mathrm{L}$ & $4.30-13.20$ \\
\hline PA & 28 & $\$ \$$ & $\mathrm{mg} / \mathrm{L}$ & $180-400$ \\
\hline HBV-DNA data & $1.41 \mathrm{E}+04$ & $\#$ & $U / L$ & $\leq 5.00 \mathrm{E}+02$ \\
\hline $\mathrm{HBsAg}$ & + & & & - \\
\hline $\mathrm{HBsAb}$ & - & & & $+/-$ \\
\hline $\mathrm{HBeAg}$ & - & & & - \\
\hline $\mathrm{HBeAb}$ & + & & & - \\
\hline $\mathrm{HBcAb}$ & + & & & - \\
\hline HBcab-IgM & + & & & - \\
\hline HCV-Ag & - & & & - \\
\hline HCV-Ab & - & & & - \\
\hline
\end{tabular}

ALT, alanine aminotransferase; AST, aspartate aminotransferase; TP, total protein; ALB, albumin; TBIL, total bilirubin; DBIL, direct bilirubin; TBA, total bile acid; GGT- $\gamma$, glutamyltransferase; CHE, cholinesterase; PA, prealbumin.

CT showed that the liver parenchyma was not uniform and patchy enhancement was observed locally (Figure 1A,B). The hepatic portal veins' left and right branches were normal, and the hepatic veins were unclear (Figure 1B). Also, the collateral circulation of the portal vein could be seen (Figure 1B).

One week after admission, we performed a biopsy of the patient's liver and performed HE staining on the pathological specimen. It was found that hepatocyte edema, patchy hemorrhagic necrosis, pseudo-lobule formation, hepatic sinusoidal dilatation and congestion, fibrous tissue hyperplasia in the portal area, and inflammatory cell infiltration. The pathological findings suggested a diagnosis of HVOD (Figure 2A,B).

During hospitalization, a treatment regimen combining
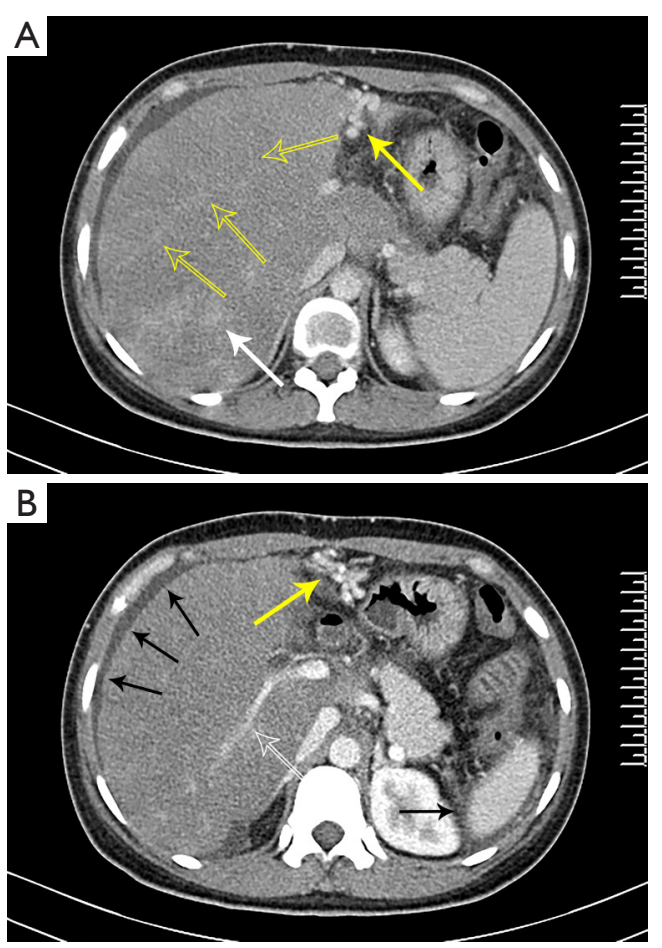

Figure 1 Patchy enhancement can be seen in the local liver parenchyma (A, white arrow). The right hepatic vein showed stenosis, and the left hepatic vein and middle hepatic vein were unclear (A, yellow unfilled arrow). Effusion is visible around the liver and spleen (B, black arrow). The collateral circulation of the portal vein can be seen (A,B, yellow arrow). The portal vein right branch shows stenosis (B, white unfilled arrow).

Chinese and Western medicine was adopted. The TCM therapy consisted of a Wei Ling decoction and Chaihu Shugan powder to tonify the spleen, invigorate blood circulation, and remove dampness through diuresis. The Western medicine therapy included the compound glycyrrhizin as well as low amounts of glutathione, levofloxacin, and spironolactone to protect the liver, decrease inflammation, and promote diuresis.

After a series of supportive treatments, the patients' clinical symptoms were significantly relieved, and she was discharged on the 90th day after admission.

After one year, the patient was re-admitted to the hospital, complaining of anorexia, fatigue, and progressive discomfort in the right flank. On physical examination, she was jaundiced, had moderately yellow sclera, showed percussion tenderness over the hepatic region, and had hepatomegaly and splenomegaly. However, on this occasion, there was no abdominal distention or tenderness and no 

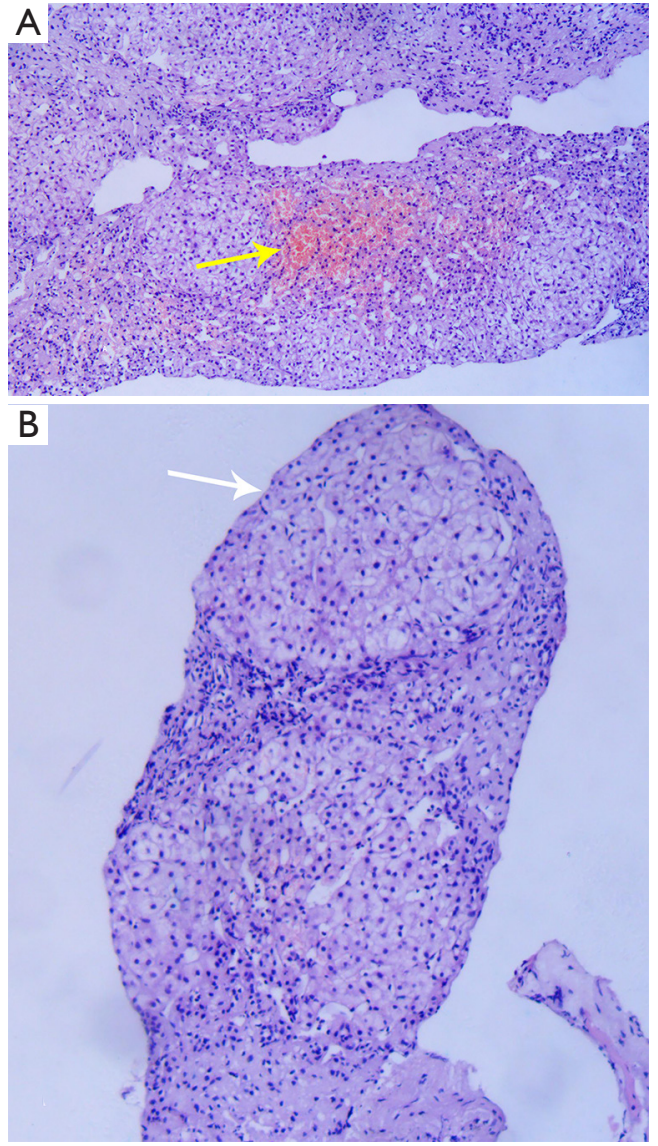

Figure 2 Hepatic sinus dilatation, partial hepatic sinus congestion (A, yellow arrow), local hepatocyte oedema, nodular formation (B, white arrow), and significant inflammatory cell infiltration can be seen.

rebound tenderness.

Laboratory tests showed that alkaline phosphatase (ALP) (297 U/L) and glutamyl transpeptidase (GGT) (190 U/L) were increased significantly (42-98 and 0-35 U/L, respectively). Levels of ALT (44 U/L) and AST (52 U/L) were also higher than normal (0-40 U/L). Total protein $(58.7 \mathrm{~g} / \mathrm{L})$ was below the normal range $(60.0-87.0 \mathrm{~g} / \mathrm{L})$, and serum prealbumin $(67 \mathrm{mg} / \mathrm{L}$, normal range $180-400 \mathrm{mg} / \mathrm{L})$ was significantly lower than at the previous hospitalization (Table 2).

The patient underwent an abdominal Doppler US examination on the first day, an abdominal CT scan on the second day of admission, and on the third day, an abdominal MRI scan was performed.

On this occasion, abdominal Doppler US examination showed an enlarged liver with a less smooth surface,
Table 2 Results of laboratory tests for the second hospitalization

\begin{tabular}{lcccc}
\hline Inspection item & $\begin{array}{c}\text { Inspection } \\
\text { result }\end{array}$ & Mark & Unit & $\begin{array}{c}\text { Reference } \\
\text { value }\end{array}$ \\
\hline ALT & 44 & $\#$ & $\mathrm{U} / \mathrm{L}$ & $0-40$ \\
AST & 52 & $\#$ & $\mathrm{U} / \mathrm{L}$ & $0-40$ \\
TP & 58.7 & $\$$ & $\mathrm{~g} / \mathrm{L}$ & $60.0-87.0$ \\
ALB & 36.3 & & $\mu \mathrm{mol} / \mathrm{L}$ & $35.0-53.0$ \\
TBIL & 55.3 & $\#$ & $\mu \mathrm{mol} / \mathrm{L}$ & $0-23.0$ \\
DBIL & 27 & $\#$ & $\mu \mathrm{mol} / \mathrm{L}$ & $0-6.8$ \\
TBA & 88.1 & $\#$ & $\mu \mathrm{mol} / \mathrm{L}$ & $0-10.0$ \\
ALP & 297 & $\#$ & $\mathrm{U} / \mathrm{L}$ & $42-96$ \\
GGT & 190 & $\#$ & $\mathrm{U} / \mathrm{L}$ & $0-35$ \\
CHE & 4.57 & & $\mathrm{KU} / \mathrm{L}$ & $4.30-13.20$ \\
PA & 67 & $\$$ & $\mathrm{mg} / \mathrm{L}$ & $180-400$ \\
ADA & 17.5 & $\#$ & $\mathrm{U} / \mathrm{L}$ & $0.0-15.0$ \\
\hline
\end{tabular}

ALT, alanine aminotransferase; AST, aspartate aminotransferase; TP, total protein; ALB, albumin; TBIL, total bilirubin; DBIL, direct bilirubin; TBA, total bile acid; ALP, alkaline phosphatase; GGT- $\gamma$, glutamyltransferase; CHE, cholinesterase; PA, prealbumin; ADA, adenosine deaminase.

enhanced echogenicity and uneven distribution of liver parenchyma, and several hypoechoic nodules. The three hepatic veins were obviously thinner and distorted than normal, and the small branches were blurred. CDFI: the blood velocity of hepatic veins was slow. The portal vein was not widened, the mean blood flow velocity of the right portal vein was $0.18 \mathrm{~m} / \mathrm{s}$, and the accessory umbilical vein was open. No stenosis or dilation was observed in the IVC, and the echo of blood flow was unobstructed.

Compared to the findings seen at the first admission, the enhanced CT images showed that the liver was as large and the liver parenchyma showed more uneven enhancement, with a large, relatively low-density area visible in the right lobe (Figure $3 A$ ). The portal vein's right branch was narrower than at the previous admission, and the hepatic veins, the left branch of the portal vein, and its branches were not clearly observed (Figure 3B). On the CT images, nodules of different sizes that were diffusely distributed in the liver parenchyma could be seen, and the degree of enhancement of the nodules was similar to that of the normal parenchyma of the left lobe (Figure $3 A, B$ ).

Abdominal MRI showed more nodules in the parenchyma of the liver than those observed during the first 

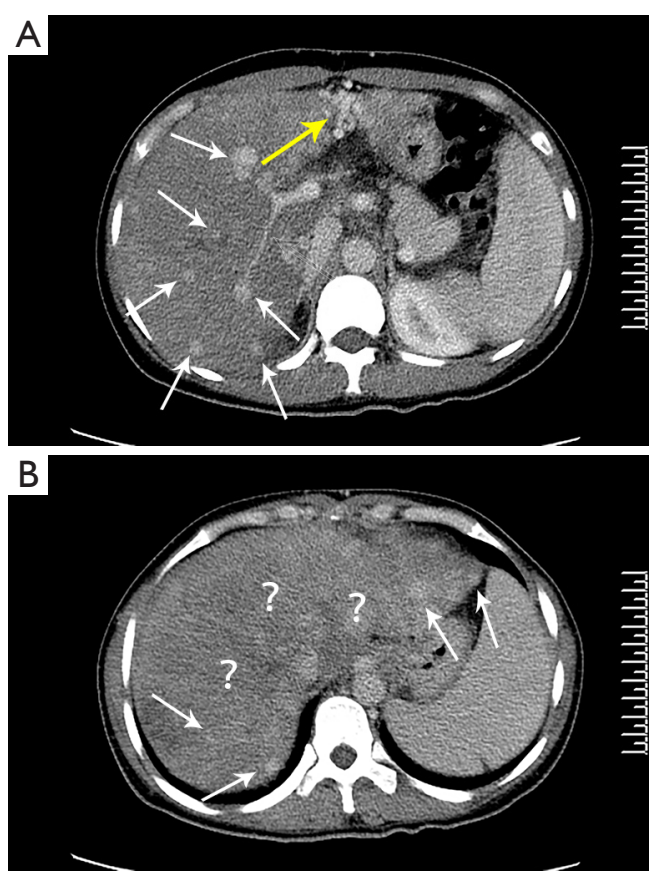

Figure 3 The right branch of the portal vein shows stenosis (A, yellow arrow). The left hepatic vein, the middle hepatic vein, the right hepatic vein, and the left branch of the portal vein, and their branches are not clearly shown (B). Nodules of different sizes are diffusely distributed in the liver parenchyma (A,B, white arrow).

admission. These nodules showed slightly increased signal intensity on T1WI and equal or slightly decreased signal intensity on T2WI (Figure 4A,B). An enhanced scan showed heterogeneous enhancement of the liver parenchyma and no intensification of the nodules, and a delayed scan showed an equal signal.

During this hospitalization, we again adopted a treatment regimen combining Chinese and Western medicine. The TCM treatment mainly contained Xiaoyao powder to soothe the liver and strengthen the spleen, acupuncture points to RiYue (GB24) and QiMen (LR14) to activate blood circulation and dissolve stasis, and an intravenous drip of tanshinone to improve microcirculation. The Western medical treatment mainly served to protect the liver and reduce high enzyme levels, and the main drug used was lowdose glutathione.

On a ninth day after admission, the symptoms of discomfort in the right flank and jaundice were relieved, and although the patient remained anorexic, she was discharged. We advised the patient to continue treatment outside the
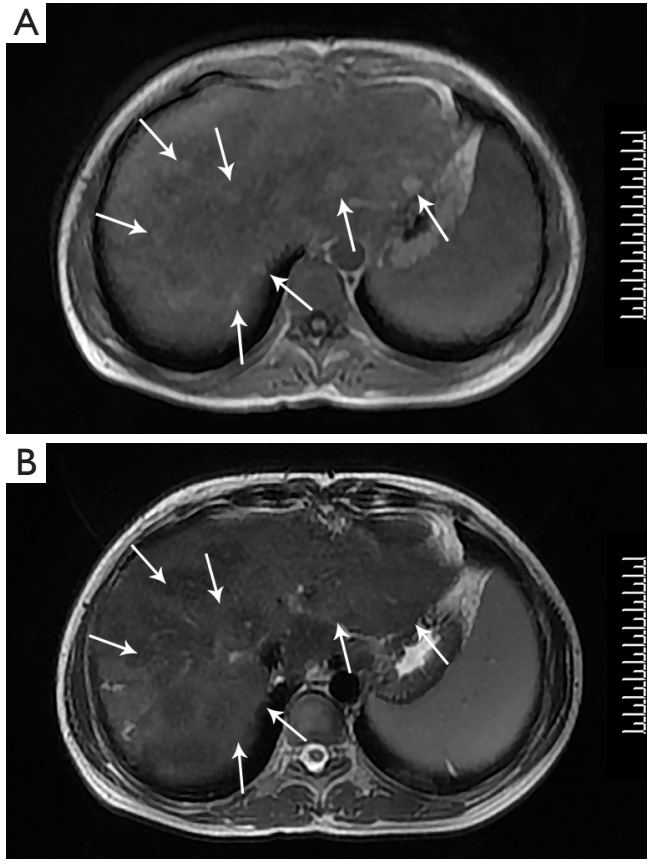

Figure 4 Nodules of different sizes are diffusely distributed in the liver parenchyma, with a slightly increased signal intensity on T1WI and an equal or slightly decreased signal intensity on T2WI (A,B, white arrow).

hospital and follow-up regularly while eating a light diet and avoiding spicy food, which echoed our recommendations after the last hospitalization. At her most recent follow-up, the patient stated that her current quality of life was good.

\section{Discussion}

Plant species containing PA are distributed worldwide. Hepatic metabolites of PA can react with cellular proteins and/or DNA, and the formation of adducts may lead to cancer, pulmonary hypertension, congenital anomalies, or liver disease $(9,12)$. Tusanqi is a commonly used TCM in China to improve blood circulation, dissipate blood stasis, and treat traumatic injuries. However, it contains $\mathrm{PA}$ in its roots, leading to HVOD if ingested excessively or improperly $(6,11)$.

The recognition of HVOD has only occurred gradually since Stuart and Bras (13) first reported the condition in 1950. HVOD is a serious disease, often presenting with clinical symptoms such as jaundice, ascites, abdominal pain, abdominal distension, hepatomegaly, splenomegaly, and hyperbilirubinemia $(14,15)$. Wu et al. (16) calculated that 
the mortality rates of severe, moderate, and mild HVOD were $98 \%, 23 \%$, and $9 \%$, respectively.

A study by Yang et al. (17) showed that the metabolites of PA in the liver directly impact hepatic sinusoidal endothelial cells. Pathological manifestations in the liver of patients with HVOD have certain characteristics, such as slight disorganization of hepatic lobules, hepatic sinuses, central lobular veins, and sublobular veins, and congestion, dilatation, and thickening of the venous wall with collagen deposition. Moreover, positive CD34 has been observed in the central veins' subendothelial space and perivenular zones of hepatic acini $(10,14,15)$.

Although treatment for HVOD patients is usually supportive, there are some reports of more effective treatments. Richardson et al. (18) reported that defibrotide had been approved for the treatment of HVOD with renal or hepatic dysfunction after HSCT in the United States and for the treatment of severe HVOD after HSCT by the EU. Lee et al. (19) showed that intravenous injection of $\mathrm{N}$-acetylcysteine (NAC) was an effective method to treat HVOD, and Takada et al. (20) showed that recombinant human soluble thrombomodulin (rTM) attenuated the clinical symptoms of HVOD by protecting liver sinusoidal endothelial cells (LSCEs) and enhancing their function. Furthermore, Luo et al. (21) showed that IVC stenting combined with direct intrahepatic portocaval shunt (DIPS) placement alleviated clinical symptoms in patients with HVOD-related decompensated cirrhosis caused by botanical hepatotoxicity.

Medical imaging examination is of great value for the diagnosis and treatment of HVOD. The common Doppler US manifestations of patients with HVOD are: (I) a diffuse increase in liver volume, dense enhancement of parenchymal echo, uneven distribution, and a "map-like" change; (II) the diameter of hepatic veins becomes thinner and blood flow velocity slows down; (III) the portal vein diameter widens, the flow velocity slows down and even the reflux Ascites, gallbladder wall edema, umbilical vein opening and other manifestations of portal hypertension (22-26). Trenker et al. (27) found that contrast-enhanced US allows assessment of the vascular supply of organs by using a purely intravascular contrast medium and contend that ultrasound contrast is helpful for the diagnosis of HVOD. In the past, many investigators considered HVOD part of BCS. The most valuable imaging feature that distinguishes BCS from HVOD is whether the main hepatic veins and IVC lumens are unobstructed. Color Doppler US combined with pulsed Doppler US can be used as a reliable method for rapid and noninvasive diagnosis of HVOD $(24,25)$.

Common CT findings of HVOD include (I) diffuse hepatomegaly with uneven reduction of liver parenchyma density on plain scan; (II) the liver parenchyma shows characteristic "map-like" and "speckled" uneven enhancement during the venous phase and the equilibrium phase, and a low-density edema zone around the portal vein appears. (III) the lumen of hepatic veins are narrow or unclear; (IV) the condition is usually associated with ascites, pleural effusion, gallbladder gastrointestinal wall edema, and other extrahepatic signs (26,28-31).

Typical MRI findings include (I) plain scan with enlarged liver volume and uneven signal. (II) the three hepatic veins are fine or indistinctly displayed; (III) T2 weighted imaging shows patchy high signal and "cloud floc" shapes. (IV) MRI dynamic contrast-enhanced scans show uneven enhancement in the arterial and venous phases, presenting a "speckle" shape, with more obvious enhancement in the delayed phase (29). In comparison with the US, contrastenhanced MRI allowed differentiation of extremely slow portal venous flow from thrombosis in one patient $(26,29,32,33)$.

It is precisely because medical imaging examinations provide us with valuable information to make an accurate diagnosis and dynamic observation of this case. When the patient was admitted for the first time, we found the liver's abnormality (especially the hepatic veins) was the cause of a series of secondary changes and clinical symptoms in the patient's abdominal cavity. Typical CT findings such as ascites, locally patchy enhancement of liver parenchyma, and the unclear display of hepatic veins helped us preliminarily determine that the patient's condition's etiology was HVOD or BCD. Doppler US confirmed the presence of color signals of blood flow in the hepatic veins, and there was no thrombosis in the vascular lumens, which provided us with vital help in diagnosing HVOD. MRI examination provided better resolution than CT. Thus, in this case, in addition to showing abnormal liver changes, the MRI examination helped determine whether the intrahepatic nodules were benign or malignant.

Our patient has been treated and followed for more than three years, and the disease has progressed from the acute to the chronic stage. In the acute stage, the patient's clinical symptoms were mainly jaundice, ascites, abdominal distension, hepatomegaly, and splenomegaly. During the chronic stage and after a series of inpatient and out-of-hospital treatments, although the patient's cirrhosis regenerative nodules increased, portal hypertension symptoms, such as ascites, were alleviated. 
We believe that this is due to the well-established collateral circulation of the portal vein, which effectively mitigates the effects of portal hypertension.

At the second admission, the CT examination showed scattered nodules of different sizes in the liver parenchyma. We consider that this imaging change was due to the synergistic effect of posterior sinus portal hypertension caused by stenosis or occlusion of the central lobular veins and sublobular veins after HVOD, and sinus portal hypertension caused by post-hepatitis B cirrhosis, which results in a significant decrease in hepatic blood flow. Therefore, the liver parenchyma background density decreases, and regenerative nodules under the liver's background become obvious.

Also, the number of regenerative nodules during the second hospitalization was significantly increased compared to those observed during the first hospitalization. We consider that the occurrence of this phenomenon was caused by hepatitis and cirrhosis and the presence of HVOD.

In conclusion, this is the first report to dynamically describe the process of HVOD from the acute to the chronic stage and is also the first report on the possible impact of HVOD on the development of cirrhosis in patients with hepatitis B. By reviewing the patient's course; we believe that HVOD may accelerate the development of cirrhosis. These findings may help radiologists improve the diagnosis of HVOD and provide some value for the prognosis of clinical treatment.

\section{Acknowledgments}

We thank Wang TongMing for his support in CT and MRI scanning and post-processing. Similarly, we would like to thank Yue DaCheng for his advice on treating this disease with the combination of Chinese and Western medicine. We are also grateful to the experts and professors in Henan Provincial Hospital's pathology department of traditional Chinese medicine and Western China Hospital, whom we have repeatedly consulted in the pathological diagnosis of this disease.

Funding: None.

\section{Footnote}

Conflicts of Interest: All authors have completed the ICMJE uniform disclosure form (available at http://dx.doi. org/10.21037/qims-20-48). The authors have no conflicts of interest to declare.

Ethical Statement: The authors are accountable for all aspects of the work in ensuring that questions related to the accuracy or integrity of any part of the work are appropriately investigated and resolved. The study was conducted in accordance with the Declaration of Helsinki (as revised in 2013). The study was approved by the Ethics Committee of Henan Province Hospital of TCM (No. E171021), and written informed consent was obtained from the patient for publication of this study and any accompanying images. A copy of the written consent is available for review by the Editor-in-Chief of this journal.

Open Access Statement: This is an Open Access article distributed in accordance with the Creative Commons Attribution-NonCommercial-NoDerivs 4.0 International License (CC BY-NC-ND 4.0), which permits the noncommercial replication and distribution of the article with the strict proviso that no changes or edits are made and the original work is properly cited (including links to both the formal publication through the relevant DOI and the license). See: https://creativecommons.org/licenses/by-nc-nd/4.0/.

\section{References}

1. Magnabosco EM, Rivera ML, Prolla IR, de Verney YM, de Mello ES. Hepatic veno-occlusive disease: report of a case. J Pediatr (Rio J) 1997;73:115-8.

2. Ka AS, Michel G, Imbert P, Diakhate I, Seye MN. Hepatic veno-occlusive disease due to dietary toxins in a five-year-old child in Senegal. Med Trop (Mars) 2006;66:514-5.

3. Kakar F, Akbarian Z, Leslie T, Mustafa ML, Watson J, van Egmond HP, Omar MF, Mofleh J. An outbreak of hepatic veno-occlusive disease in Western afghanistan associated with exposure to wheat flour contaminated with pyrrolizidine alkaloids. J Toxicol 2010;2010:313280.

4. Bane A, Seboxa T, Mesfin G, Ali A, Tsegaye Y, W/ Tensae M, G/Selassie S, Haile T. An outbreak of venoocclusive liver disease in northern Ethiopia, clinical findings. Ethiop Med J 2012;50:9-16.

5. Schneider J, Tsegaye Y, W/Tensae M, G/Selassie S, Haile T, Bane A, Ali A, Mesfin G, Seboxa T. Venoocclusive liver disease: a case report. Ethiop Med J 2012;50:47-51.

6. Wang X, Qi X, Guo X. Tusanqi-Related Sinusoidal 
Obstruction Syndrome in China: A Systematic Review of the Literatures. Medicine 2015;94:e942.

7. Sun Z, Kang J, Zhang Y. Hepatic veno-occlusive disease related to Gynura segetum: A case report. Medicine 2018;97:e0552.

8. Yang M, Ruan J, Gao H, Li N, Ma J, Xue J, Ye Y, Fu PP, Wang J, Lin G. First evidence of pyrrolizidine alkaloid $\mathrm{N}$-oxide-induced hepatic sinusoidal obstruction syndrome in humans. Arch Toxicol 2017;91:3913-25.

9. Edgar JA, Molyneux RJ, Colegate SM. Pyrrolizidine Alkaloids: Potential Role in the Etiology of Cancers, Pulmonary Hypertension, Congenital Anomalies, and Liver Disease. Chem Res Toxicol 2015;28:4-20.

10. Lin G, Wang JY, Li N, Li M, Gao H, Ji Y, Zhang F, Wang H, Zhou Y, Ye Y, Xu HX, Zheng J. Hepatic sinusoidal obstruction syndrome associated with consumption of Gynura segetum. J Hepatol 2011;54:666-73.

11. Wang JY, Gao H. Tusanqi and hepatic sinusoidal obstruction syndrome. J Dig Dis 2014;15:105-7.

12. Ruan J, Gao H, Li N, Xue J, Chen J, Ke CQ, Ye Y, Fu PP, Zheng J, Wang J, Lin G. Blood Pyrrole-Protein Adducts--A Biomarker of Pyrrolizidine AlkaloidInduced Liver Injury in Humans. J Environ Sci Health C Environ Carcinog Ecotoxicol Rev 2015;33:404-21.

13. Stuart KL, Bras G. Veno-occlusive disease of the liver in Barbados; case reports. West Indian Med J 1956;5:33-6.

14. Gao H, Li N, Wang JY, Zhang SC, Lin G. Definitive diagnosis of hepatic sinusoidal obstruction syndrome induced by pyrrolizidine alkaloids. J Dig Dis 2012;13:33-9.

15. Dai N, Yu YC, Ren TH, Wu JG, Jiang Y, Shen LG, Zhang J. Gynura root induces hepatic veno-occlusive disease: A case report and review of the literature. World J Gastroenterol 2007;13:1628-31.

16. Wu XW, Wang WQ, Liu B, Xu JM, Yu YQ, Zhang S, Shen $Y$. Hepatic veno-occlusive disease after taking Gynura Rhizome: The value of multidetector computed tomography in diagnosing the disease and evaluating the clinical therapeutic effect. Hepatol Res 2012;42:304-9.

17. Yang M, Ruan JQ, Fu PP, Lin G. Cytotoxicity of pyrrolizidine alkaloid in human hepatic parenchymal and sinusoidal endothelial cells: Firm evidence for the reactive metabolites mediated pyrrolizidine alkaloid-induced hepatotoxicity. Chem Biol Interact 2016;243:119-26.

18. Richardson PG, Grupp SA, Pagliuca A, Krishnan A, Ho VT, Corbacioglu S. Defibrotide for the treatment of hepatic veno-occlusive disease/sinusoidal obstruction syndrome with multiorgan failure. Int J Hematol Oncol 2017;6:75-93.

19. Lee AC, Aung L. Treatment of hepatic veno-occlusive disease in children with $\mathrm{N}$-acetylcysteine. Pediatr Blood Cancer 2019;66:e27518.

20. Takada S, Miyashita T, Yamamoto Y, Kanou S, Munesue S, Ohbatake Y, Nakanuma S, Okamoto K, Sakai S, Kinoshita J, Makino I, Nakamura K, Tajima H, Takamura H, Ninomiya I, Fushida S, Ohta T. Soluble Thrombomodulin Attenuates Endothelial Cell Damage in Hepatic Sinusoidal Obstruction Syndrome. In Vivo 2018;32:1409-17.

21. Luo S, Chu J, Huang H, Yao K. Direct Intrahepatic Portocaval Shunt for Sinusoidal Obstruction Syndrome Associated with Hepatotoxicity of Pyrrolizidine Alkaloids. Biomed Res Int 2018;2018:9804582.

22. Schiavetti A, Matrunola M, Varrasso G, Padula A, Castello MA. Ultrasound in the management of hepatic veno-occlusive disease in three children treated with dactinomycin and vincristine. Pediatr Hematol Oncol 1996;13:521-9.

23. Zieger MM, Koscielniak E. Diagnosis and follow-up of veno-occlusive disease of the liver by use of Doppler ultrasound. Pediatr Radiol 1993;23:137-9.

24. Deeg KH, Glöckel U, Richter R, Beck J. Diagnosis of veno-occlusive disease of the liver by color-coded Doppler sonography. Pediatr Radiol 1993;23:134-6.

25. Chan SS, Colecchia A, Duarte RF, Bonifazi F, Ravaioli F, Bourhis JH. Imaging in Hepatic Veno-occlusive Disease/Sinusoidal Obstruction Syndrome. Biol Blood Marrow Transplant 2020;26:1770-9.

26. Zhang Y, Yan YL, Song B. Noninvasive imaging diagnosis of sinusoidal obstruction syndrome: a pictorial review. Insights Imaging 2019;10:110.

27. Trenker C, Sohlbach K, Dietrich CF, Gorg C. Clinical diagnosis of veno-occlusive disease using contrast enhanced ultrasound. Bone Marrow Transplant 2018;53:1369-71.

28. Yang S, Wu J, Lei S. CT Features of Hepatic Venoocclusive Disease: A Meta-analysis. Acad Radiol 2018;25:328-37.

29. Zhou H, Wang YX, Lou HY, Xu XJ, Zhang MM. Hepatic sinusoidal obstruction syndrome caused by herbal medicine: CT and MRI features. Korean J Radiol 2014;15:218-25.

30. Shao H, Chen HZ, Zhu JS, Ruan B, Zhang ZQ, Lin $\mathrm{X}$, Gan MF. Computed tomography findings of hepatic 
veno-occlusive disease caused by Sedum aizoon with histopathological correlation. Braz J Med Biol Res 2015;48:1145-50.

31. Kan X,F Ye J, Rong XX, Lu ZW, Li X, Wang Y, Yang L, Xu KS, Song YH, Hou XH. Diagnostic performance of Contrast-enhanced CT in Pyrrolizidine Alkaloidsinduced Hepatic Sinusoidal Obstructive Syndrome. Sci Rep 2016;6:37998.

Cite this article as: Li B, Gao F, Liu X, Pan J, Liu L. Herbal tea-induced hepatic veno-occlusive disease in China: a case description. Quant Imaging Med Surg 2021;11(8):3882-3889. doi: 10.21037/qims-20-48
32. van den Bosch MA, van Hoe L. MR imaging findings in two patients with hepatic veno-occlusive disease following bone marrow transplantation. Eur Radiol 2000;10:1290-3.

33. Mortele KJ, Van Vlierberghe H, Wiesner W, Ros PR. Hepatic veno-occlusive disease: MRI findings. Abdom Imaging 2002;27:523-6. 